\title{
SERIES EXPANSIONS IN TERMS OF THE TEMPERATURE FUNCTIONS OF PORITSKY AND POWELL*
}

\author{
BY \\ D. V. WIDDER \\ Harvard University
}

1. Introduction. In a paper by $H$. Poritsky and R. A. Powell [1] certain solutions $T_{n}(x, t), n=0,1,2, \cdots$, of the heat equation

$$
\frac{\partial^{2} u(x, t)}{\partial x^{2}}=\frac{\partial u(x, t)}{\partial t}
$$

were defined. Explicitly, for $x>0$ and $t>0$

$$
T_{n}(x, t)=2 \int_{0}^{t} k(x, t-y) \frac{y^{n}}{n !} d y,
$$

where $k(x, t)$ is the fundamental solution

$$
k(x, t)=(4 \pi t)^{-1 / 2} \exp \left(-x^{2} / 4 t\right), \quad t>0 .
$$

Those authors show that

$$
T_{n}(0, t)=\frac{t^{n+1 / 2} 4^{n+1}(n+1) !}{\pi^{1 / 2}(2 n+2) !},
$$

so that $T_{n}(x, t)$ may also be expressed by the integral

$$
T_{n}(x, t)=\int_{0}^{t} h(x, t-y) T_{n}(0, y) d y,
$$

where

$$
h(x, t)=\frac{x}{t} k(x, t)=-2 \frac{\partial}{\partial x} k(x, t), \quad t>0 .
$$

Thus $T_{n}(x, t)$ may be interpreted as the temperature of a semi-infinite bar (along the positive $x$-axis), initially $(t=0)$ at zero temperature, the finite end of which is maintained at temperatures given by the function (1.3).

We define here the related functions

$$
U_{n}(x, t)=\int_{0}^{t} h(x, t-y) \frac{y^{n}}{n !} d y, \quad x>0, \quad t>0,
$$

and inquire what solutions of the heat equation (1.1) can be expanded in series of the form

$$
u(x, t)=\sum_{n=0}^{\infty}\left\{a_{n} T_{n}(x, t)+b_{n} U_{n}(x, t)\right\} .
$$

*Received July 10, 1961. This work was supported (in part) by the Air Force Office of Scientific Research, under Contract AF 49(638)-574. 
We are able to find necessary and sufficient conditions of several types which will insure the validity of such expansions. For example, Eq. (1.6) holds for $0<t<r$ if and only if

$$
u(x, t)=\int_{0}^{\infty} k(x+y, t) f(y) d y,
$$

where $f(y)$ is an entire function of growth $(2,1 /(4 r))$. See Sec. 3 for the definition of such functions.

2. Properties of $T_{n}(x, t)$ and $U_{n}(x, t)$. Let us first find the Laplace transforms of $T_{n}(x, t)$ and $U_{n}(x, t)$ with respect to the variable $t$. We prove

Theorem 1. For $x>0, \operatorname{Re} s=\sigma>0$

$$
\begin{aligned}
& \int_{0}^{\infty} \exp (-s t) T_{n}(x, t) d t=\exp \left(-x s^{1 / 2}\right) / s^{n+3 / 2}, \\
& \int_{0}^{\infty} \exp (-s t) U_{n}(x, t) d t=\exp \left(-x s^{1 / 2}\right) / s^{n+1}
\end{aligned}
$$

Since $T_{n}$ and $U_{n}$ are defined as convolutions, the above transforms are obtained by multiplication of the following:

$$
\int_{0}^{\infty} \exp (-s t) h(x, t) d t=\exp \left(-x s^{1 / 2}\right), \quad x>0, \quad \sigma>0
$$

(see p. 288 of [3]),

$$
\begin{array}{ll}
\int_{0}^{\infty} \exp (-s t) t^{n+1 / 2} d t=\frac{\Gamma(n+3 / 2)}{s^{n+3 / 2}}, & \sigma>0, \\
\int_{0}^{\infty} \exp (-s t) t^{n} d t=\frac{n !}{s^{n+1}}, & \sigma>0 .
\end{array}
$$

The absolute convergence of all integrals involved insures the validity of the composition theorem.

Thus far the functions $T_{n}$ and $U_{n}$ are defined for positive $x$ only. We now define them for negative $x$ by analytic continuation. Clearly this analytic continuation is not given by the integrals (1.4) and (1.5) since $h(x, t)$ is an odd function of $x$ and since, as a consequence, the functions defined by those integrals suffer discontinuities at $x=0$. We proceed by replacing (1.4) and (1.5) by other integrals of equal value for $x>0$ but which are analytic for $-\infty<x<\infty$.

Theorem 2. For $x>0, t>0$

$$
\begin{aligned}
& T_{n}(x, t)=2 \int_{0}^{\infty} k(x+y, t) \frac{y^{2 n+1}}{(2 n+1) !} d y \\
& U_{n}(x, t)=2 \int_{0}^{\infty} k(x+y, t) \frac{y^{2 n}}{(2 n) !} d y .
\end{aligned}
$$

The proofs of the two formulas are similar, so that we may confine attention to (2.3). By the uniqueness theorem for Laplace transforms it will be sufficient to show that both sides of Eq. (2.3) have the same transform. For the left hand side we already have the 
result in Eq. (2.1). For the right hand side we may use the known transform (formula (6.2) of [3])

$$
\int_{0}^{\infty} \exp (-s t) k(x, t) d t=\frac{\exp \left(-x s^{1 / 2}\right)}{2 s^{1 / 2}}, \quad 0<\sigma<\infty, \quad 0<x<\infty .
$$

Thus

$$
\begin{aligned}
2 \int_{0}^{\infty} \exp (-s t) d t \int_{0}^{\infty} k(x+y, t) & \frac{y^{2 n+1}}{(2 n+1) !} d y \\
& =\int_{0}^{\infty} \frac{y^{2 n+1}}{(2 n+1) !} \frac{\exp \left[-(x+y) s^{1 / 2}\right]}{s^{1 / 2}} d y=\frac{\exp \left(-x s^{1 / 2}\right)}{s^{n+3 / 2}} .
\end{aligned}
$$

The interchange in the order of integration is justified by Fubini's theorem, the integrand of the integral (2.3) being positive.

Since the integrals (2.3) and (2.4) are familiar Weierstrass transforms they may now be used to define $T_{n}(x, t)$ and $U_{n}(x, t)$ as solutions of Eq. (1.1) for all real $x$. (See, for example, Theorem 3.3 of [4]).

3. Entire functions. For the reader's convenience, we recall here some facts about entire functions in a form in which we shall use them. An entire function $f(z)$ is said to be of growth $(\rho, \sigma)$ if it is of order $\leqq \rho$. In addition it is stipulated that when the equality holds, then the type of $f(z)$ is $\leqq \sigma$. For example, the functions $1, z$, sinh $z$, cosh $3 z^{2}, z$ $\exp z^{2}$ are all of growth $(2,3)$. A function $f(z)$ of growth $(\rho, \sigma)$ satisfies for every $\epsilon>0$ the relation

$$
f(z)=0\left(\exp (\sigma+\epsilon)|z|^{\rho}\right), \quad|z| \rightarrow \infty
$$

Let

$$
f(z)=\sum_{n=0}^{\infty} \frac{c_{n}}{n !} z^{n}, \quad f^{*}(z)=\sum_{n=0}^{\infty} \frac{\left|c_{n}\right|}{n !} z^{n} .
$$

The rate of growth of either function can be characterized in terms of $\left|c_{n}\right|$. The results needed are:

Theorem 3.1. The functions (3.1) are of growth $(2, \sigma)$ if and only if

$$
\limsup _{n \rightarrow \infty}\left|c_{n}\right|^{2 / n} / n \leqq 2 \sigma / e .
$$

Theorem 3.2. The functions (3.1) are of order 1 and of type $\sigma$ if and only if

$$
\limsup _{n \rightarrow \infty}\left|c_{n}\right|^{1 / n}=\sigma \text {. }
$$

4. The principal expansion criterion. We treat expansion in terms of the sets $T_{n}(x, t)$ and $U_{n}(x, t)$ separately. The main theorem will then be obtained by combining the two results.

Theorem 4.1. A function $u(x, t)$ has the expansion

$$
u(x, t)=\sum_{n=0}^{\infty} a_{n} T_{n}(x, t),
$$

convergent for $0<t<r$, if and only if

$$
u(x, t)=\int_{0}^{\infty} k(x+y, t) g(y) d y, \quad 0<t<r,
$$


where $g(y)$ is an odd entire function of growth $(2,1 / 4 r)$ defined by the series

$$
g(y)=2 \sum_{n=0}^{\infty} \frac{a_{n} y^{2 n+1}}{(2 n+1) !} .
$$

We prove first the sufficiency of the condition (4.2), (4.3). If it is satisfied, substitution of the series (4.3) in the integral (4.2) formally gives

$$
u(x, t)=\sum_{n=0}^{\infty} a_{n} T_{n}(x, t) .
$$

Here we have used Eq. (2.3). Since $T_{n}(x, t)$ is positive for $t>0$ the term-by-term integration will be valid if

$$
\int_{0}^{\infty} k(x+y, t) g^{*}(y) d y<\infty .
$$

Here $g^{*}(y)$ is obtained from $g(y)$ by changing $a_{n}$ in (4.3) to $\left|a_{n}\right|$. Since $g(y)$, and hence $g^{*}(y)$, has growth $(2,1 / 4 r)$ we have for any positive $\theta$ less than 1

$$
g^{*}(y)=0\left(\exp \left[y^{2} /(4 r \theta)\right]\right), \quad y \rightarrow \infty .
$$

Since $k(x+y, t)$ behaves, as $y \rightarrow \infty$, essentially like exp $\left(-y^{2} / 4 t\right)$ it is clear from (4.5) that the inequality (4.4) holds for any positive $t<r$. This completes the proof of the sufficiency of the condition.

Conversely, assume the expansion (4.1). In particular we have for $x=0$ from (1.3) that

$$
u(0, t)=4(t / \pi)^{1 / 2} \sum_{n=0}^{\infty} \frac{(n+1) ! a_{n}(4 t)^{n}}{(2 n+2) !} .
$$

Since this series is assumed to converge for $0<t<r$ we have by Stirling's formula that

$$
\limsup _{n \rightarrow \infty}\left|a_{n}\right|^{1 / n} / n \leqq 1 /(e r) .
$$

From this inequality we can infer by use of (3.2) that $g(y)$ and $g^{*}(y)$, as defined by (4.3), have growth $(2,1 /(4 r)$. Since only odd powers of $y$ are involved we may replace $n$ by $2 n$ and $b_{2 n}$ by $a_{n}$ in formula (3.2). It then becomes (4.6) with $\sigma=1 /(4 r)$. And now the formal integration of series (4.3) effected in the earlier part of this proof again becomes valid for $0<t<r$ under the present assumptions. This completes the proof.

The companion result for expansions in terms of the $U_{n}(x, t)$ is the following.

Theorem 4.2. A function $u(x, t)$ has the expansion

$$
u(x, t)=\sum_{n=0}^{\infty} b_{n} U_{n}(x, t),
$$

convergent for $0<t<r$, if and only if

$$
u(x, t)=\int_{0}^{\infty} k(x+y, t) h(y) d y, \quad 0<t<r,
$$

where $h(y)$ is an even entire function of growth $(2,1 /(4 r)$ defined by the series

$$
h(y)=2 \sum_{n=0}^{\infty} \frac{b_{n} y^{2 n}}{(2 n) !} \text {. }
$$


The proof is similar to that for Theorem 4.1 and is omitted.

By combining the two foregoing theorems we obtain the main result.

Theorem 4.3. A function $u(x, t)$ has an expansion

$$
u(x, t)=\sum_{n=0}^{\infty}\left\{a_{n} T_{n}(x, t)+b_{n} U_{n}(x, t)\right\},
$$

convergent for $0<t<r$, if and only if

$$
u(x, t)=\int_{0}^{\infty} k(x+y, t) f(y) d y, \quad 0<t<r,
$$

where $f(y)$ is entire of growth $(2,1 / 4 r)$. Here

$$
2 a_{n}=f^{(2 n+1)}(0), \quad 2 b_{n}=f^{(2 n)}(0), \quad n=0,1,2, \cdots .
$$

It is clear that the sum of a series (4.10) must satisfy (1.1) for $0<t<r$, and that $u(x, 0+)=0(0<x<\infty), u(x, 0+)=f(-x)(-\infty<x<0)$,

$$
u(0, t)=\frac{1}{(\pi t)^{1 / 2}} \sum_{n=0}^{\infty} \frac{a_{n}(n+1) !(4 t)^{n+1}}{(2 n+2) !}+\sum_{n=0}^{\infty} \frac{b_{n} t^{n}}{n !} .
$$

5. Expansions valid for $0<t<\infty$. Let us restrict attention in this section to expansions in terms of the functions $U_{n}(x, t)$. Analogous considerations would apply to series in the $T_{n}(x, t)$.

Theorem 5.1. A function $u(x, t)$ has an expansion (4.7) convergent for $0<t<\infty$ and with

$$
\limsup _{n \rightarrow \infty}\left|b_{n}\right|^{1 / n}=q^{2}
$$

if and only if

$$
u(x, t)=\int_{0}^{\infty} k(x+y, t) h(y) d y, \quad 0<t<\infty,
$$

where $h(y)$ is an even entire function of order 1 and type $q$ defined by the series (4.9).

As in Theorem 4.1 the proof depends on the validity of the term-by-term integration of series (4.9) after multiplication by $k(x+y, t)$. This process is clearly valid for $0<t<\infty$ if $h^{*}(y)$ is of order 1. In one-half of the theorem this is part of the hypothesis. In the other half it is a consequence of (5.1). For, since series (4.9) involves only even powers of $y$ we may replace $n$ by $2 n$ in formula (3.3), with $c_{2 n}=b_{n}$. Then (5.1) implies

$$
\limsup _{n \rightarrow \infty}\left|b_{n}\right|^{1 / 2 n}=q
$$

and the proof is complete.

The criterion for the validity of the expansion (4.7), (5.1) may also be given a neat form in terms of the Laplace transform of $u(x, t)$.

Theorem 5.2. A function $u(x, t)$ has an expansion (4.7) convergent for $0<t<\infty$, $0<x<\infty$, and with

$$
\underset{n \rightarrow \infty}{\limsup }\left|b_{n}\right|^{1 / n}=q
$$


if and only if for $x>0, \operatorname{Re} s>0$,

$$
\int_{0}^{\infty} \exp (-s t) u(x, t) d t=\exp \left(-s x^{1 / 2}\right) F(s),
$$

where $F(s)$ is analytic for $|s|>q$, not for $|s| \geqq q$, and is defined by the series

$$
F(s)=\sum_{n=0}^{\infty} \frac{b_{n}}{s^{n+1}}
$$

Assume first Eq. (4.7), the coefficients satisfying (5.2). Multiply both sides by exp - st and integrate with respect to $t$. By (2.2) the result is (5.3), (5.4) provided

$$
\int_{0}^{\infty} \exp (-\sigma t) \sum_{n=0}^{\infty}\left|b_{n}\right| U_{n}(x, t) d t<\infty
$$

$(\sigma=\operatorname{Re} s)$. Here we have used the fact the $U_{n}(x, t) \geqq 0$. Equivalently we may show that

$$
\sum_{n=0}^{\infty} \frac{\left|b_{n}\right|}{\sigma^{n+1}}<\infty .
$$

This is clearly true for $\sigma>q$ by (5.2). The analytic character of $F(s)$ follows from the fact that the radius of convergence of the power series $\sum b_{n} z^{n}$ is precisely $1 / q$ by virtue of the hypothesis (5.2).

Conversely, assume Eq. (5.3). Then (5.2) also follows from the analytic character of $F(s)$. It is a familiar fact that $F(s)$ is the Laplace transform of the function

$$
F(t)=\sum_{n=0}^{\infty} \frac{b_{n} n^{n}}{n !} \text {. }
$$

Moreover, when $x>0, \exp -x \sqrt{s}$ is the transform of $h(x, t)$. That is, the product on the right of (5.3) is the transform of the convolution of the function $F(t)$ with $h(x, t)$. By the uniqueness theorem for Laplace transforms

$$
u(x, t)=\int_{0}^{t} h(x, t-y) F(y) d y .
$$

Now substitute the series (5.5) in the integral (5.6) and use the definition (1.5) to obtain

$$
u(x, t)=\sum_{n=0}^{\infty} b_{n} U_{n}(x, t) .
$$

The formal substitution will be valid if

$$
\sum_{n=0}^{\infty}\left|b_{n}\right| \int_{0}^{t} h(x, t-y) \frac{y^{n}}{n !} d y=\sum_{n=0}^{\infty}\left|b_{n}\right| U_{n}(x, t)<\infty .
$$

But for $0<x<\infty, 0<t<\infty$

$$
0<U_{n}(x, t)<\frac{t^{n}}{n !} \int_{0}^{t} h(x, t-y) d y=\frac{t^{n}}{n !} \operatorname{erfc}\left[x /(4 t)^{1 / 2}\right],
$$

so that the series (5.7) is dominated by one which converges by (5.2).

Since the condition (5.3) restricts the function $u(x, t)$ only for $x>0$, the series (4.3) cannot be expected, in the first instance, to define $u(x, t)$ for $x<0$. But the terms of the 
series are defined for $x<0$, and the series does in fact converge there, so that $u(x, t)$ could be defined by analytic continuation for negative $x$.

6. Examples. Let us illustrate the three principal expansion theorems by examples. For Theorem 4.2 choose

$$
\begin{gathered}
u(x, t)=\frac{\exp \left[A x^{2} /(1-4 A t)\right]}{(1-4 A t)^{1 / 2}} \operatorname{erfc} x[4 t(1-4 A t)]^{-1 / 2} . \\
u(0, t)=(1-4 A t)^{-1 / 2}=\sum_{n=0}^{\infty}\left(\begin{array}{c}
2 n \\
n
\end{array}\right)(A t)^{n} \\
b_{n}=A^{n}(2 n) ! / n ! \\
h(y)=2 \sum_{n=0}^{\infty} \frac{A^{n} y^{2 n}}{(2 n) !}=2 \exp \left(A y^{2}\right) .
\end{gathered}
$$

We see by inspection that $h(y)$ has growth $(2, A)$ and that the power series for $u(0, t)$ converges for $|t|<1 / 4 A$ as predicted by the theorem. This example shows that the integral (4.8) may converge in a larger region than the series (4.7). If $A<0$ the integral converges for all positive $t$ whereas the series (6.1), and hence (4.7), diverges for $t=1 /(4 A)$.

For Theorem 5.1 choose $b_{n}=b^{2 n}, q=b>0$. Then

$$
h(y)=2 \cosh b y, \quad u(0, t)=\exp \left(b^{2} t\right), \quad b>0 .
$$

Clearly the function $h(y)$ is of order 1 and type $b$, as predicted. The function $u(x, t)$ is known explicitly (see p. 45, Eq. (9) of [5]):

where

$$
u(x, t)=\frac{1}{2}\left\{v_{b}(x, t)+v_{-b}(x, t)\right\} \exp \left(b^{2} t\right),
$$

$$
v_{b}(x, t)=\exp (b x) \operatorname{erfc}\left(\frac{x}{(4 t)^{1 / 2}}+b t^{1 / 2}\right) .
$$

Finally, to illustrate Theorem 5.2 we may compute the Laplace transform of the function (6.2).

From formula (12), p. 177 of [6] we have

$$
\int_{0}^{\infty} \exp (-s t) \exp \left(b^{2} t\right) v_{b}(x, t) d t=\frac{\exp \left(-b x-x s^{1 / 2}\right)}{\left(s+b s^{1 / 2}\right)} .
$$

Replace $b$ by $-b$ in this formula and thus by addition obtain

$$
\int_{0}^{\infty} \exp (-s t) u(x, t) d t=\frac{\exp \left(-x s^{1 / 2}\right)}{s-b^{2}}=\exp \left(-x s^{1 / 2}\right) \sum_{n=0}^{\infty} \frac{b^{2 n}}{s^{n+1}} .
$$

Here $q=b^{2}$, and $F(s)$ is analytic for $|s|>b^{2}$.

\section{REFERENCES}

1. H. Poritsky and R. A. Powell, Certain solutions of the heat conduction equation, Quart. Appl. Math. 18, 97-106 (1960)

2. R. P. Boas, Entire functions, Academic Press, 1954

3. D. V. Widder. Integral transforms related to heat conduction, Annali di Matematica 42, 279-305 (1956)

4. I. I. Hirschman and D. V. Widder, The convolution transform, Princeton University Press, 1955

5. H. S. Carslaw and J. C. Jaeger, Conduction of heat in solids, Clarendon Press, 1948

6. Staff of the Bateman Manuscript Project, Tables of integral transforms, vol. 1, McGraw-Hill Book Co., 1954 